\title{
Application of Interview Method on the Design of Assistive Device - Power-Assisted Exoskeleton for Caregivers as the Example
}

\author{
*Yu-Sheng Huang ${ }^{1}$, Yi-Ren Song ${ }^{2}$, and Po-Wen Hsueh ${ }^{3}$ \\ ${ }^{1}$ National Kaohsiung University of Science and Technology Industrial Design \\ Master Class Student, Kaohsiung, Taiwan \\ ${ }^{2}$ National Kaohsiung University of Science and Technology Industrial Design \\ Master Class Associate Professor, Kaohsiung, Taiwan \\ ${ }^{3}$ National Kaohsiung University of Science and Technology Department of Mechanical and Automation \\ Engineering Master Class Associate Professor, Kaohsiung, Taiwan
}

\begin{abstract}
The burden on caregivers is increasing because of the rapidly population growth. Therefore, power-assisted exoskeleton become an important assistive device to the caregivers for reducing the risk of permanent injury during the elder care process. Recently, most of commercial power-assisted exoskeleton are designed for the entertainment, industrial, medical, and military applications. However, majority of users are not able to accept the kind of products after considering to the factor of self-estimate, because using the power-assisted exoskeleton will make them look different from normal people. Hence, this paper presents the novel design concept of the power-assisted exoskeleton according to the 60 potential users' interviews and the design psychology of Donald A. Norman. The result shows that the power-assisted exoskeletons are necessary with the features of Chromatology, Product design, Technological and Mechanism, and so on, that would be acceptable to the caregivers. The substance of the study redesigns a power-assisted exoskeleton base on these features to induce the interests of the caregivers. It can make more people accept using power-assisted exoskeleton rather than keep their bias from assistive device, thus decreasing the injury incur while working.
\end{abstract}

Keywords: power-assisted exoskeleton; design; caregiver; mechanism; self-estimate 